\title{
LIPID DISORDERS: ADVANCES IN RESEARCH AND CHALLENGES IN CLINICAL PRACTICE
}

\author{
Carlos A. Aguilar-Salinas ${ }^{1,2,3}$, Teresa Tussié-Luna ${ }^{4}$ and Paloma Almeda-Valdés ${ }^{1,2}$ \\ ${ }^{1}$ Department of Endocrinology and Metabolism; ${ }^{2}$ Metabolic Disorders Research Unit, Instituto Nacional de Ciencias \\ Médicas y Nutrición Salvador Zubirán, Mexico City; ${ }^{3}$ Tec Salud, Instituto Tecnológico y de Estudios Superiores de \\ Monterrey, Monterrey, N.L.; ${ }^{4}$ Molecular Biology and Genomic Medicine Unit, Instituto Nacional de Ciencias Médicas \\ y Nutrición Salvador Zubirán, Mexico City, México.
}

Research in lipoprotein metabolism has been a highly successful endeavor ${ }^{1}$. This topic has been among the primary targets for the application of the newest research techniques, leading up to the rapid development of innovative products, international consortia, and multidisciplinary interventions ${ }^{2}$. In addition, translation of basic science into clinical practice has been very efficient. The high prevalence of lipid disorders, the large contribution of these conditions in the pathogenesis of cardiovascular morbidity, and the availability of potent lipid-lowering agents have been determinants of the large impact of these studies in our societies. Despite these remarkable progress, there are large differences in the implementation of the best available evidence to prevent cardiovascular events through correction of plasma lipids concentration among countries ${ }^{3}$. In this special issue of the Revista de Investigación Clínica - Clinical and Translational Investigation - we collect five review articles that will not only allow the readers to update their knowledge about new research and therapeutic approaches but also to identify the main clinical challenges to be faced in the majority of the low- and medium-income countries.

\section{Corresponding author:}

*Carlos A. Aguilar-Salinas

Departamento de Endocrinología y Metabolismo

Instituto Nacional de Ciencias Médicas y Nutrición

Salvador Zubirán

Vasco de Quiroga, 15

Col. Belisario Domínguez Sección XVI, Del. Tlalpan

C.P. 14080, Mexico City, Mexico

E-mail: caguilarsalinas@yahoo.com
An example of a frontline research in lipid disorders is presented by Alejandra Rodríguez and Päivi Pajukanta from UCLA, USA. Pajükanta's group has made significant contributions in the pathophysiology of primary hyperlipidemias ${ }^{4}$. The application of the systems biology approach to search for new regulatory pathways of lipoprotein metabolism is described in their review. On the other hand, an state of the art review on the use of new technologies to treat dyslipidemias is comprehensively presented by Carlos Olimpo Mendivil, who is a clinical investigator with a highly productive research career ${ }^{5}$.

We draw attention to the readers in two topics that need an attitude change in many clinicians. First, dyslipidemia in patients with type 2 diabetes is among the most important targets to reduce the diabetesrelated complications ${ }^{6}$. However, this opportunity is missed in a large number of cases treated in primary care centers. Prof. Klaus G. Parhofer, from the University of Munich, Germany, and a highly recognized expert in Europe and member of the writing committees of several European lipid-related guidelines ${ }^{7}$, reviews the controversial aspects of this topic and proposes

Received for publication: 31-08-2018 Approved for publication: 03-09-2018 doi: 10.24875/RIC.M18000001 
specific actions to increase the number of cases under control. Second, familial combined hyperlipidemia is the most common primary dyslipidemia in western countries. However, the relevance of this condition is poorly recognized in the guidelines and by primary care physicians. Dr. Ivette Cruz-Bautista and her group from the Lipid Clinic of the Instituto Nacional de Ciencias Medicas y Nutrición, describe the current knowledge about this common condition. Information is presented in highly comprehensive manner; this article will help to clinicians to recognize this condition and the high cardiovascular risk associated.

Mexicans have a high prevalence of lipid disorders. The evidence collected in the past two decades about the epidemiology, genetics, and treatment of these conditions is summarized in the review by RivasGomez and colleagues. In this article, we make a call for action to improve the access of care for patients with the lipid disorders associated with the highest cardiovascular risk (i.e., familial hypercholesterolemia and familial combined hyperlipidemia in type 2 diabetes).
We would like to express our gratitude to $\mathrm{Dr}$. Alfredo Ulloa-Aguirre, Editor-in-Chief of the Revista de Investigación Clínica - Clinical and Translational Investigation - for the invitation to act as Guest Editors for this thematic issue of the journal.

\section{REFERENCES}

1. Jeff JM, Peloso GM, Do R. What can we learn about lipoprotein metabolism and coronary heart disease from studying rare variants? Curr Opin Lipidol. 2016;27:99-104

2. Ellis KL, Hooper AJ, Burnett JR, Watts GF. Progress in the care of common inherited atherogenic disorders of apolipoprotein B metabolism. Nat Rev Endocrinol. 2016;12:467-84.

3. Danchin N, Almahmeed W, Al-Rasadi K, et al. Achievement of low-density lipoprotein cholesterol goals in 18 countries outside Western Europe: The international choLesterol management practice study (ICLPS). Eur J Prev Cardiol. 2018;25:1087-94.

4. Ripatti P, Rämö JT, Söderlund S, et al. The Contribution of GWAS loci in familial dyslipidemias. PLoS Genet. 2016;12:e1006078.

5. Singh SA, Andraski AB, Pieper B, et al. Multiple apolipoprotein kinetics measured in human HDL by high-resolution/accurate mass parallel reaction monitoring. J Lipid Res. 2016;57:714-28.

6. Chait A, Goldberg I. Treatment of dyslipidemia in diabetes: recent advances and remaining questions. Curr Diab Rep. 2017;17:112.

7. Hegele RA, Ginsberg HN, Chapman MJ, et al. The polygenic nature of hypertriglyceridaemia: implications for definition, diagnosis, and management. Lancet Diabetes Endocrinol. 2014;2:655-66. 